\title{
Solar cycle variations of the internal structure and dynamics of the sun
}

\author{
H. Shibahashi \\ Department of Astronomy, University of Tokyo, Tokyo 113-0033, Japan \\ email: shibahashi@astron.s.u-tokyo.ac.jp
}

\begin{abstract}
Helioseismic analysis of solar global oscillations allows investigation of the internal structure and dynamics of the sun. I summarize the recent observational results, putting emphasis on the bands of slower- and faster-than-average rotation migrating in latitude over the solar cycle in a manner similar to the zones of solar activity. I also review theoretical considerations so far proposed as a cause of the periodic oscillation of the rotation profile.
\end{abstract}

\section{Introduction}

One of the very important issues in solar physics is the solar cycle with a period of 22 years, which manifests itself in many ways associated with magnetic activities such as semi-cyclic variation in sunspot numbers, flare activities, a flip-flop change of the magnetic polarity, and so on. The solar cycle is believed to be caused by a dynamo mechanism acting near the base of the convection zone, but the details are not yet been well known. Prior to the development of the methods of helioseismology, the solar surface was the only place where we could observe, and various theoretical investigations of a dynamo action, which were made with various assumptions concerning the invisible solar interior, were checked only by those surface phenomena. The situation has changed with the development of helioseismology, which has provided a new tool to see the invisible interior of the sun. Some changes in the solar internal structure and dynamics associated with the solar cycle may be expected to be found with the help of helioseismology.

A frequency change over the solar cycle has indeed been detected. However, it has not yet clarified the change in the thermal structure near the base of the convection zone which is believed to be the most plausible seat of the solar dynamo. The situation is a little different for investigation of the dynamics in the convection zone. Careful analyses of the ground-based observations of the solar surface rotation have shown that alternate zones of fast and slow rotation originate at mid-latitude regions and drift to the equator in 22 years; this fact was confirmed by recent analyses of helioseismic data. In this review, I summarize development of observations of such solar cycle variations of rotation, and then discuss the cause of the observed periodic oscillation of the rotation profile.

\section{Overview of observational studies of torsional oscillations}

\subsection{Ground-based studies of the solar surface rotation}

Howard \& LaBonte (1980) are the first who discovered the presence of alternate zones of fast and slow rotation drifting from mid-latitudes to the equator in 22 years. They found these by analyzing the full-disk Dopplergram data using the $\lambda 5250.2$ (Fe I) line taken with the 150-foot tower telescope at Mt. Wilson in 1966 Jan - 1979 Dec. At a given latitude, the rotation rate cyclically changes. If we observe this from the rotating frame with the averaged rotation rate of the sun, the given latitudinal zone shifts cyclically eastward 
and westward. Howard \& LaBonte (1980) thought that this rotation pattern represented a periodic torsional motion about the solar rotation axis and hence named the observed phenomenon 'torsional oscillations'. The main characteristics of this phenomenon are as follows: (i) Alternate zones of fast and slow rotation originate at the mid-latitude regions and drift to the equator in 22 years. (ii) The pattern is almost symmetric about the equator. (iii) The phases of the bands in the two hemispheres are almost identical. (iv) The amplitude $\sim 3 \mathrm{~ms}^{-1}$ is not instantaneously correlated with the magnetic activity. (v) The solar activity zones are centered on the poleward boundary of a fast zone. (vi) The equator itself is always centered in a slow zone. Further study showed that the magnetic activity of the cycle tended to maximize in the region where the torsional wave nearest the equator enhanced the shear (LaBonte \& Howard 1982). Two other different approaches were also used to uncover the torsional pattern from the same data set mentioned above (Snodgrass 1985; Ulrich et al. 1988). Furthermore, it was shown that a torsional oscillation pattern is present in the rotation of the photospheric magnetic field (Snodgrass 1991). Although the magnetic rotation torsional pattern is not identical to the Doppler counterpart, it is similar enough to provide confirmation.

Direct Doppler measurements have been continued at Mt. Wilson. Improvement in the measuring system made the data taken there after late 1985 more useable for studies of the large-scale velocities. Ulrich $(1998,2001)$ analyzed the data taken in 1986 - 2000, and described the following additional characteristics of the torsional oscillations: (i) During the early phase of the magnetic activity cycle, the latitude of the zonal velocity marks a lower boundary of the magnetic active zone. (ii) During the magnetic maximum phase, the torsional oscillation pattern is not well defined. (iii) The torsional oscillation pattern does not seem to be connected as a single and unified phenomenon. (iv) The polar regions exhibit substantial changes in their average rotation rate. (v) The magnetic field reversal occurs at the time when the polar speed-up is most evident. These characteristics are clearly seen in Fig. 1, which is the synoptic plots of solar velocity and magnetic fields. By carrying out auto-correlation analyses, Ulrich (2001) found that the torsional oscillations contain a set of long-lived components having azimuthal structure described by functions of the form $e^{i m \phi}$ where $m$ is $1<m \leqslant 8$ and $\phi$ denotes the azimuthal angle.

\subsection{Helioseismic observations of the torsional oscillations}

The solar internal rotation can be deduced from helioseismology through the analyses of the rotational splitting of frequencies. Nonradial f-modes are essentially the surface waves which are sensitive to the depth of $\sim R_{\odot} / \ell$, where $\ell$ denotes the spherical degree of the mode, hence they are suitable to be used to confirm that the surface torsional oscillation extends beneath the photosphere. Kosovichev \& Schou (1997) analyzed a 144-d dataset taken with MDI on SOHO and first succeeded in detecting the zonal shear flows beneath the Sun's surface from the rotational splitting of f-modes with $\ell=120-250$. Schou (1999) extended the analyses of the f-mode frequency splitting data taken with MDI. He used 12 72-d datasets taken in 1996 May - July and 1999 Feb - April, and showed that the zonal flows migrate from mid-latitude to low latitude beneath the photospheric level. His result also implied that the presence of high latitude zonal flow migrating poleward. This tendency was later confirmed by other helioseismic studies (e.g. Antia \& Basu 2001). Helioseismic investigation using p-modes as well as f-modes makes it possible to see the deeper zone. Schou et al. (1998) carried out the inversion of the rotational frequency splitting of acoustic modes with a medium- $\ell$ set $(\ell \leqslant 250)$ taken with MDI, and showed that alternating zonal bands of slightly faster and slower rotation are superposed on the relatively smooth latitudinal variation in the rotational profile and that these bands reach to the depth of about $5 \%$ of the solar radius. 


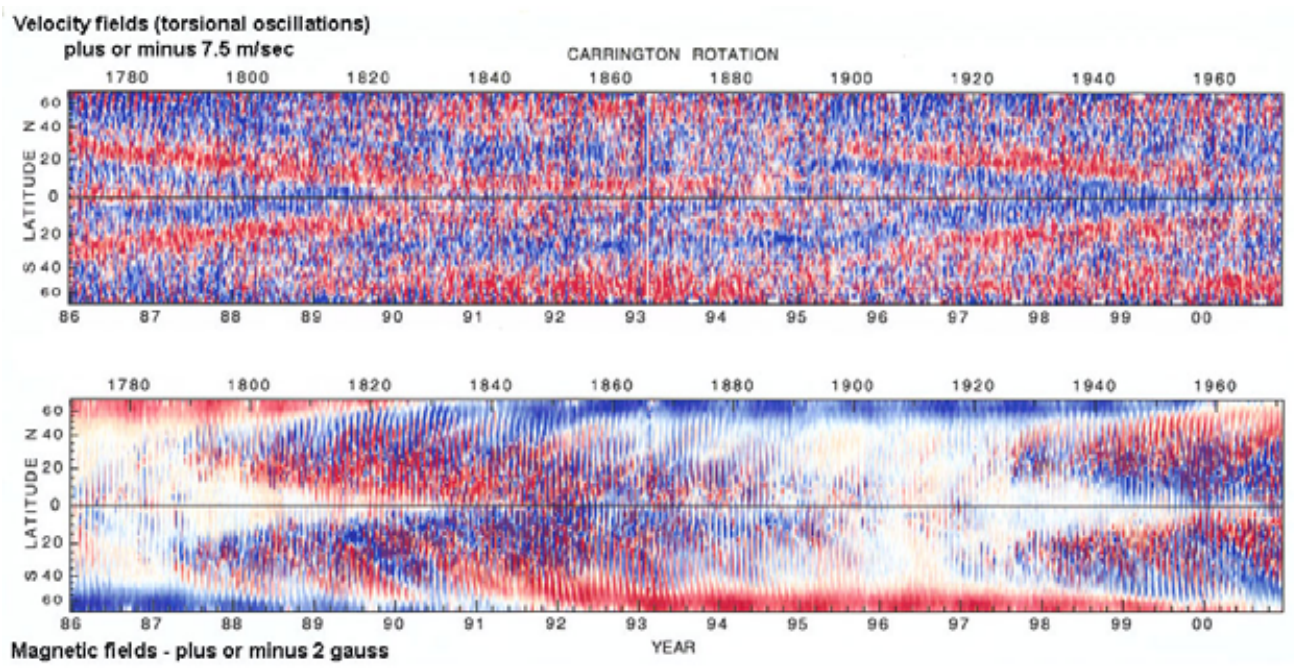

Figure 1. Synoptic plots of azimuthal velocity residual (upper) and magnetic field of the sun (lower) based on the data taken with the 150-foot tower telescope at Mt. Wilson. The original plots are seen on a Web page, http://www.astro.ucla.edu/ ${ }^{\sim}$ obs/torsional.html. By courtesy of R. K. Ulrich.

With the increase of the observational time span, investigation of the long-term features of the torsional oscillations became possible. Howe et al. (2000a) established, using complementary helioseismic observations over 4 yr from the GONG network (1995 May 1999 June) and from the MDI instrument on board SOHO (1996 May - 1998 June, 1998 Oct - Dec, $1999 \mathrm{Feb}$ - Nov) that the banded flows extend downward at least $60 \mathrm{Mm}$ and thus are evident over a significant fraction of the solar convection zone. These bands at mid-latitude were also clearly shown to move equatorward. At high latitudes, variations are more dynamical than at lower latitudes. Probably this is related to the lesser moment of inertia. Howe et al. (2000a) also noticed that the evolving zonal bands are present at high latitudes even before the prominent large-scale magnetic eruptions begin. Antia \& Basu (2000), who analyzed independently the same 4-yr GONG data, reached similar conclusions. By comparing the deduced rotation rate as a function of depth and latitude with its time average, they showed that the magnitude of the zonal flow increases with solar activity, whereas such increases are not seen in the pattern when the mean of the full rotation rate is subtracted. They also concluded that at all latitudes there appears to be a tendency of the bands of faster or slower rotation to move up with time.

Howe et al. (2000b) continued their analyses by analyzing the differences between the rotation profile and its time average. They claimed these differences seem anti-correlated across the base of the convection zone, which may imply angular momentum exchange across the tachocline. From frequency analysis, they further claimed that at the base of the convection zone the rotation profile seems to be varying with a 1.3 -yr periodicity. However, Antia \& Basu (2000) found no evidence for such a periodic variation from the same data. In this situation, it may be fair to say that the presence of a 1.3-yr periodic variation at the base of the convection zone is still controversial (cf. Toomre et al. 2003). The claim of discovery of a 1.3-yr variation has already stimulated some theoretical investigations concerning the interaction between gravity waves and the differential rotation in the tachocline (Kim \& MacGregor 2001, 2003; Talon, Kumar, \& Zahn 2002; Wolff \& Mayr 2004). Komm et al. (2003) derived the angular momentum as a function of radius and time with the help of the rotation profile resulting from the GONG and the MDI 


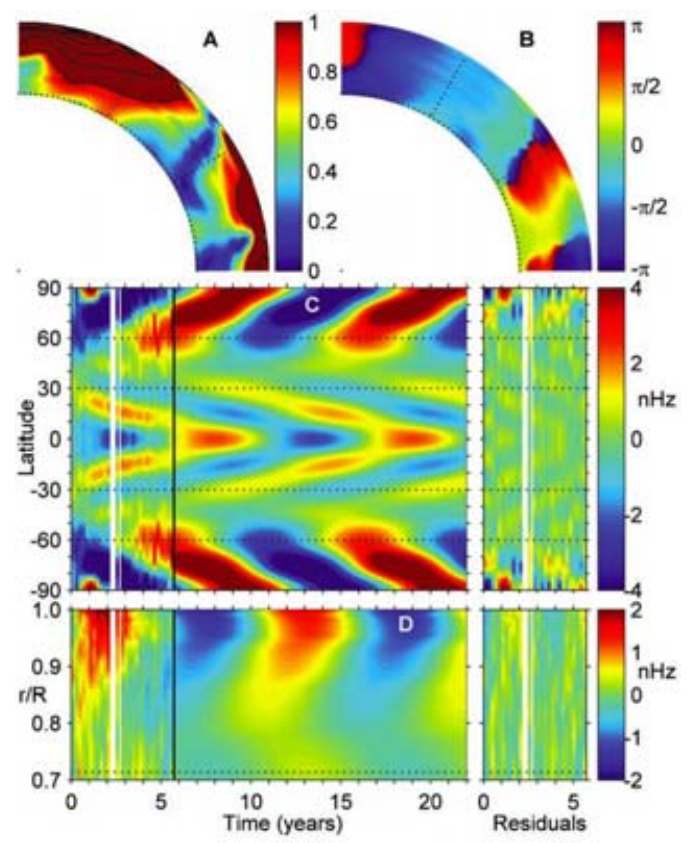

Figure 2. Results of Vorontsov et al. (2002). (A) Amplitude and (B) phase obtained by fitting a sinusoidal function with an 11-yr period to the variation of internal rotation. The phase is defined to be zero at the solar minimum. (C) The rotational variation as a function of time and latitude at $r / R_{\odot}=0.98$ are shown for the first six years, and thereafter the time series is continued by exhibiting the 11-yr harmonic fit. (D) As for (C), but showing the rotation variation at latitude $20^{\circ}$ as a function of depth. Adapted from Vorontsov et al. (2002).

data, and found that the base of the convection zone can be identified as a local maximum in relative angular momentum after subtracting the contributions of the solid-body rotation. According to them, the angular momentum shows the strongest time variation near the tachocline, and this variation is related to the $1.3-\mathrm{yr}$ periodicity found by Howe et al. (2000b).

Analyses of the 27 sets of 72-d data taken by MDI on SOHO during 1996 May - 2002 Jan were made by Vorontsov et al. (2002), who performed 2D differential inversion of the rotational splitting data with a 360-d dataset as a reference. According to their results, the low-latitude equatorward migrating feature is likely to be limited to the upper $30 \%$ of the convection zone. On the other hand, acceleration of the rotation seen in latitudes higher than $60^{\circ}$ was found to penetrate much deeper, and it reaches up to the base of the convection zone. Similar results were obtained by Basu \& Antia (2003), who also analyzed the 28 sets of 72-d MDI data taken during 1996 May - 2002 Mar as well as 62 sets of 108-d data from GONG covering 1996 May - 2001 July. No prominent, well-structured variation was found by Vorontsov et al. (2002) and by Basu \& Antia (2003) near the base of the convection zone, contrary to the results of Howe et al. (2000b). Vorontsov et al. (2002) and Basu \& Antia (2003) fitted a harmonic function of an 11-yr periodicity to the observational data, and from the residual they suggested the possible presence of an 11/3-yr periodicity. This component may be expected from the nonsinusoidal form of the temporal variation. It could also arise if the basic period is different from $11 \mathrm{yr}$.

Apart from global helioseismology, by which the solar global eigenfrequencies are measured, a new trend is development of local helioseismology. One successful method is time-distance helioseismology, by which the acoustic-wave travel times from a certain 
point on the solar surface to other points are measured. The travel time is dependent on the flow along the ray path of the wave, hence we can get information concerning the local velocity field in the sun. This technique was applied by Giles, Duvall, \& Scherrer (1998) to measure the subsurface rotation and meridional flow. Using the data taken in the summer of 1996 by the MDI, they confirmed the presence of bands of faster-thanaverage and slower-than-average rotation, and showed that the most significant change occurs at high latitudes. Methods of local helioseismology do not assume symmetry with respect to the equatorial plane, so the analyses based on these methods are sensitive to north-south asymmetries in the flows. Giles et al. (1998) indeed found a difference in the zonal flow between the northern and southern hemispheres. Time-distance helioseismology analysis was applied by Beck, Gizon, \& Duvall (2002) to the longer time-spanned MDI data covering 1996 May - 2001 July with a gap from 1998 June until 1999 March. They found that the meridional flow is strongly antisymmetric as a function of latitude, and that the peak velocity is about $15 \mathrm{~m} \mathrm{~s}^{-1}$ toward the pole at mid-latitudes, going to zero near the equator. They also clearly showed that the torsional patterns migrate from mid-latitude toward the equator over the solar cycle. The torsional oscillation is faster equatorward of the mean activity latitude and slower poleward. Furthermore, the time-varying component of the meridional flow was found to correlate very well with the torsional oscillations, with a more strongly poleward flow corresponding to a slower rotation. Hence, it is clear that there is a strong link between the time-varying component of the meridional flow and the torsional oscillations. By carrying out time-distance analyses at many sites on the solar disk, we can obtain synoptic maps of subsurface plasma flow. Zhao \& Kosovichev (2004) applied this technique to the MDI data covering the years 1996 - 2002 to obtain the subsurface plasma-flow at depths of $0-12 \mathrm{Mm}$. The results for the zonal flows agree well with previous results. It should be pointed out that meridional flows of the order of $20 \mathrm{~m} \mathrm{~s}^{-1}$ were found to remain poleward during the whole period of the observations.

Another powerful technique for local helioseismology is ring-diagram analysis, by which the oscillation power spectrum is obtained as a function of frequency and two-dimensional horizontal wavenumbers. In the presence of flows, acoustic waves with the same horizontal wavenumber propagating in opposite directions have their frequencies split by the Doppler effect. Such frequency splittings are used in ring-diagram analysis to measure flow velocities averaged over the depths where the modes have significant amplitude. Haber et al. (2000, 2002) succeeded in detecting meridional flow within the upper convection zone and torsional oscillations from the MDI-data taken during 1996 - 2001 with the help of ring-diagram analyses. In their synoptic map corresponding to a period of low magnetic activity, a low-order undulation in longitude with azimuthal orders $m$ ranging from 4 to 6 coexists with global-scale flows. Haber et al. (2002) suggest that this flow may be an inertial wave response which was pointed out by Ulrich (2001). As for the helioseismic investigations on the local velocity fields, readers should also consult Gizon (2004).

\section{Theoretical considerations concerning the torsional oscillation}

The equation of motion in the presence of a velocity field and a magnetic field in a rotating frame of angular velocity $\Omega$ is given in the MHD approach by

$$
\rho\left\{\frac{\partial \boldsymbol{v}}{\partial t}+(\boldsymbol{v} \cdot \boldsymbol{\nabla}) \boldsymbol{v}\right\}=-\boldsymbol{\nabla} p+\rho \boldsymbol{g}_{\mathrm{eff}}+2 \rho \boldsymbol{v} \times \boldsymbol{\Omega}+\frac{1}{\mu}(\boldsymbol{\nabla} \times \boldsymbol{B}) \times \boldsymbol{B}+\nu \boldsymbol{\nabla}^{2} \boldsymbol{v}
$$


where $\rho$ denotes the density, $p$ the pressure, $\boldsymbol{v}$ the fluid velocity, $\boldsymbol{g}_{\text {eff }}$ the effective gravity (including the centrifugal force), $\boldsymbol{\Omega}$ the angular velocity, $\boldsymbol{B}$ the magnetic field, $\boldsymbol{\mu}$ the permeability, $\nu$ the kinematic viscosity. The zonal flow of the sun is described by the azimuthal component of equation (3.1). The obvious connection of the torsional oscillation with the solar magnetic cycle, and its relatively small amplitude, suggest that the torsional oscillation is a secondary effect caused by the magnetic fields.

\subsection{Explanation in terms of forced oscillation driven by the Lorentz force}

Soon after the discovery of the torsional oscillation was reported by Howard \& LaBonte (1980), Schüssler (1981) and Yoshimura (1981) proposed independently a hypothesis that the observed torsional oscillations are driven as a feed-back of the magnetic field by the longitudinal component of the Lorentz force of the dynamo waves which simulate the observed solar magnetic cycle. They averaged the physical quantities over longitude, and separated, in the inertial frame in which $\Omega=0$ in equation (3.1), the azimuthal component of the velocity $\overline{\boldsymbol{v}_{\phi}}$ into a basic component which is determined by the processes without the effects of the Lorentz force, and a perturbed part, $\overline{\boldsymbol{v}_{\phi}^{\prime}}$, where overbars denote averaging over longitude. Then the perturbed part is approximately governed by

$$
\frac{\partial \overline{\boldsymbol{v}_{\phi}^{\prime}}}{\partial t} \simeq \frac{1}{\mu \rho} \overline{[(\boldsymbol{\nabla} \times \boldsymbol{B}) \times \boldsymbol{B}]_{\phi}}+\nu \nabla^{2} \overline{\boldsymbol{v}_{\phi}^{\prime}},
$$

where the subscript $\phi$ means the azimuthal component. The mean magnetic field in the sun is divided into two components: a poloidal component $\overline{\boldsymbol{B}_{\mathrm{p}}}$ and a toroidal component $\overline{\boldsymbol{B}_{\mathrm{t}}}$, which are varying with a period $\sim 22 \mathrm{yr}$ and the amplitudes of the order of $1 \mathrm{G}$ and $100 \mathrm{G}$, respectively. Then the azimuthal component of the Lorentz force term in equation (3.2) is given by $\left(\boldsymbol{\nabla} \times \overline{\boldsymbol{B}_{\mathrm{t}}}\right) \times \overline{\boldsymbol{B}_{\mathrm{p}}}$, which consists of a non-oscillatory part and an oscillatory part with a period one-half of the original magnetic period, and equation (3.2) can be regarded as an equation for the forced oscillation. If we take typical values for $\rho$ and consider a subsurface zone with a depth of $d \sim 0.1 R_{\odot}$, then the amplitude of the forced oscillation $\overline{\boldsymbol{v}_{\phi}^{\prime}}$ is estimated to be of the same order of magnitude of the observed torsional oscillation. A phase lag between the Lorentz force and the torsional wave is induced by the viscous term, and then the observed phase lag is explained by tuning the viscosity $\nu / d^{2}$. Yoshimura (1981) adopted his dynamo model for the observed solar magnetic cycle. According to his model, the non-oscillatory part of the Lorentz force remains in the deep regions of the convection zone and only the wave part emerges near the surface so that the forced oscillation is confined near the surface. Rüdiger et al. (1986) also discussed the torsional oscillation from a similar point of view.

The equation of motion (3.1) should be supplemented by the induction equation, which couples again the velocity field and the magnetic field. These two equations should be solved simultaneously to give strict consistency. In most cases of dynamo theories, which are often called the kinematic dynamo, as a first approximation the induction equation is usually solved first with a given form of the rotation profile and the dynamo action. Prior to development of helioseismology, the depth variation of the rotation rate was not known but was adjusted as one of the free parameters in the dynamo theories to give the right butterfly diagram of sunspots. Helioseismology made it possible to deduce the profile of the solar internal rotation as a function of depth and latitude, for the outer regions $r>0.6 R_{\odot}$ except for the high latitude regions (e.g. Thompson et al. 1996; Schou et al. 1998). The rotation profile within the convection zone was found to be different from prior expectations which had been assumed in the dynamo hypotheses. In this situation, within the kinematic dynamo models, the zero-order velocity in the induction equation should be chosen to be consistent with the helioseismic data. Covas et al. (2000) investigated 
a dynamo model in this way. In the mean field dynamo approach the magnetic field is separated into two parts: a mean field $\bar{B}$ and a fluctuating part $\boldsymbol{B}^{\prime}$. Likewise the velocity is separated into mean and fluctuating parts: $\boldsymbol{v}=\overline{\boldsymbol{v}}+\boldsymbol{v}^{\prime}$. The induction equation for the mean field is described as

$$
\frac{\partial \overline{\boldsymbol{B}}}{\partial t}=\boldsymbol{\nabla} \times\left(\overline{\boldsymbol{v}} \times \overline{\boldsymbol{B}}+\boldsymbol{E}_{\mathrm{t}}\right)+\eta \boldsymbol{\nabla}^{2} \overline{\boldsymbol{B}},
$$

where $\eta$ denotes the magnetic diffusivity. Here $\boldsymbol{E}_{\mathrm{t}}$ is defined by $\boldsymbol{E}_{\mathrm{t}} \equiv \overline{\boldsymbol{v}^{\prime} \times \boldsymbol{B}^{\prime}}$; it represents the electric field generated by fluctuations and is called the turbulent electromotive force. In principle $\boldsymbol{E}_{\mathrm{t}}$ must be calculated in terms of $\overline{\boldsymbol{B}}$ by solving the induction equation for the fluctuating part $\boldsymbol{B}^{\prime}$. That is too difficult in general, but inspection of the induction equation for the mean field (3.3) and its counterpart for the fluctuating part shows that there must be a linear relationship between $\overline{\boldsymbol{B}}$ and $\boldsymbol{B}^{\prime}$ for prescribed $\boldsymbol{v}^{\prime}$, and hence also between $\boldsymbol{E}_{\mathrm{t}}$ and $\overline{\boldsymbol{B}}$. Covas et al. (2000) adopted this standard form of the turbulent electromotive force used in the so-called $\alpha-\omega$ dynamo, and set $\boldsymbol{E}_{\mathrm{t}}=\alpha \overline{\boldsymbol{B}}$, where $\alpha$ represents the dynamo action. By tuning the functional forms of the parameters $\alpha$ and $\eta$ in equation (3.3), so that the model produces butterfly diagrams being in qualitative agreement with the observations, they showed that the torsional oscillation is induced as a feed-back from the Lorentz force. Parameter dependence of their model, as well as the influence of density stratification, were investigated by Covas, Tavakol, \& Moss (2001, 2004).

The seat of the dynamo is not yet known. The introduction of magnetic buoyancy introduced by Parker (1975) tends to suggest that a toroidal magnetic field of the strength observed in bipolar magnetic regions is not properly amplified in the convection zone, but instead has to be generated near the tachocline. However, the regeneration there of a poloidal magnetic field from the toroidal field is fraught difficulties, since the toroidal magnetic field is large enough to inhibit the fluid motions that are essential for the existence of the $\alpha$-effect. The $\alpha$-effect might operate slightly above the tachocline, or, alternatively, it could operate at the surface. Durney (2000) investigated the latter case, and compared the resultant Lorentz force as a function of time and latitude with the observed torsional oscillations. However, agreement has not yet been reached.

\subsection{Effects of cross-helicity dynamo on the velocity field}

Yoshizawa (1990) pointed out that the electromotive force should be expressed in terms of not only the mean field and the mean current but also the mean vorticity $\overline{\boldsymbol{\omega}} \equiv \boldsymbol{\nabla} \times \overline{\boldsymbol{v}}$;

$$
\boldsymbol{E}_{\mathrm{t}}=\alpha \overline{\boldsymbol{B}}-\beta \overline{\boldsymbol{J}}+\gamma \overline{\boldsymbol{\omega}} .
$$

The term $\gamma \overline{\boldsymbol{\omega}}$ can generate a seed magnetic field, and the importance of this term has been discussed in detail (e.g. Blackman \& Chou 1997; Brandenburg \& Urpin 1998; Blackman 2000). The coupling coefficient is called the cross-helicity. Itoh et al. (2004) paid attention the effect of cross-helicity on the mean velocity field. From equation (3.4) into (3.3), under the approximation of dropping $\overline{\boldsymbol{v}} \times \overline{\boldsymbol{B}}$, the quasi-stationary state of $\overline{\boldsymbol{B}}$ may occur through the condition $\overline{\boldsymbol{J}} \simeq \beta^{-1}(\alpha \overline{\boldsymbol{B}}+\gamma \overline{\boldsymbol{\omega}})$. Substituting this form into the perturbed part of equation (3.1) and taking the curl of the resultant equation, we obtain

$$
\frac{\partial \boldsymbol{\omega}^{\prime}}{\partial t} \simeq \boldsymbol{\nabla} \times\left[\nu \nabla^{2}\{\overline{\boldsymbol{v}}-(\gamma \overline{\boldsymbol{B}} / \beta)\}\right] .
$$

The term $\boldsymbol{\nabla} \times\left(\nu \nabla^{2} \overline{\boldsymbol{v}}\right)$ is the viscous term by which the vorticity tends to decay, while the term $\boldsymbol{\nabla} \times\left\{\nu \nabla^{2}(\gamma \overline{\boldsymbol{B}} / \beta)\right\}$ generates the velocity so that $\boldsymbol{v}^{\prime}$ tends to be parallel to $\overline{\boldsymbol{B}}$. Itoh et al. (2004) discuss the possibility of a periodic change of solar differential rotation 
due to cyclic change of the mean magnetic field. The observed phase lag between the torsional oscillations and the magnetic fields is explained by the viscosity.

\subsection{Explanation in terms of the magnetic quenching of the Reynolds stress}

In the solar convection zone, the turbulent viscosity may be anisotropic owing to the rotation and stratification. Although viscous diffusion tends to suppress velocity gradients, it can induce a meridional circulation if the viscosity is anisotropic, and the resultant circulation can in turn drive a differential rotation. This may be one of the possible ways to explain the differential rotation in the solar convection zone. The Reynolds stress tensor in MHD flows is split into the two parts, the diffusive part and the non-diffusive part. The latter does not vanish even for the case of uniform rotation and is essential for maintaining the differential rotation. This property is called the ' $\Lambda$-effect' (Rüdiger 1989). With the magnetic field varying with the solar cycle the stress tensors may also change with time. Küker, Rüdiger, \& Pipin (1996) considered a possibility that the $\Lambda$-effect itself is quenched by the magnetic field as a function of the ratio between the magnetic field intensity and the turbulence energy. They derived an expression for the $\Lambda$-effect, and by tuning the values of some parameters they showed that the observed torsional oscillation can be reproduced. Following this concept, Kitchatinov et al. (1999) discussed the phase relation between the torsional oscillation and the magnetic cycle. By assuming more phenomenologically the effect of the magnetic fields on viscosity, Petrovay \& Forgács-Dajka (2002) demonstrated a torsional oscillation can be induced.

\subsection{Torsional oscillation is a signature of giant cells?}

The existence of giant cells as the fundamental mode of solar convection has long been proposed on theoretical grounds, but attempts to detect them observationally have been unsuccessful. Snodgrass \& Wilson (1987) proposed an interpretation of torsional oscillations as a signature of giant cells. Upflow motion due to convection should reduce the mean angular velocity of the local region as a consequence of angular momentum conservation. In particular, poleward meridional flows initiated at a region of upflow should produce an increase in angular velocity. Conversely, equatorward flow should similarly reduce the angular velocity. Hence, if regions of upflow and downflow are localized at different latitudes, a velocity shear would develop at or near a latitude of significant downflow. By assuming that cells take the form of a system of large-scale latitude and time-dependent azimuthal convective rolls and that they propagate from the polar regions to the equator in a solar cycle, Snodgrass \& Wilson (1987) tried to explain phenomenologically the observed torsional oscillations (cf. Howard 1987). Recent development of local helioseismology shows, however, that meridional flow in a global sense does not change the sign in each of the hemispheres, and then the giant-cell hypothesis seems to be ruled out.

\subsection{Explanation of torsional oscillation as a geostrophic flow}

Spruit (2003) paid attention to the fact that horizontal flows on length scales like those of the observed torsional oscillation are dominated by the Coriolis force. If the characteristic time scale $\tau$ of the flow is long, there should be an approximate balance between the horizontal pressure gradient and the Coriolis force. This situation is called the 'geostrophic balance,' which is well known to be the case for the large-scale flows in the Earth's atmosphere and oceans. By taking as the characteristic time scale $\tau$ the time for the pattern to change noticeably due to its drift in latitude, and by estimating it to be of the order of 1 yr, Spruit (2003) then concluded that the geostrophic balance is a good approximation. 
The latitudinal component of this balance is

$$
\frac{1}{r} \frac{\partial p}{\partial \theta}=-2 \rho v_{\phi} \Omega \cos \theta
$$

where $\theta$ denotes the colatitude. According to the interpretation of the oscillation as a geostrophic flow, equation (3.6) means that the sign of the torsional oscillation detected in mid-latitude zones, with an increased rotation rate on the leading and a reduced rate on the trailing side of the magnetic activity belts, indicates that the magnetic activity belts are zones of slightly lower pressure, thus of slightly lower temperature. Spruit (2003) argues that at any given horizontal surface the temperature in the magnetic structure is lower than average. According to him, that the active regions look nevertheless brighter is explained by the fact that the opacity in the structure is reduced and then radiation is emitted from geometrically deeper layers than average, where the temperature is higher. By taking the order of the width of the belt of activity as the characteristic length, which is $\sim 3 \times 10^{8} \mathrm{~m}$, and as an estimate of $p / \rho \simeq 10^{8} \mathrm{~m}^{2} \mathrm{~s}^{-2}$, we can expect a zonal flow $v_{\phi}$ having an amplitude of the order of $\simeq 5 \mathrm{~ms}^{-1}$ with a small relative temperature change $\delta T / T$ is of the order of $10^{-5}$. This model predicts also the depth dependence of the flow, the nonaxisymmetric structure, the accompanied meridional flow and downflow.

\section{Summary}

With development of helioseismology, it became certain that the zones of faster and slower rotation extend deeply in the convection zone and that they apparently migrate equatorward and poleward, depending on their latitudes, in the solar cycle. Investigation of this flow in the convection zone is helpful for ultimately understanding of the solar cycle. This is particularly true if this flow is indeed the forced oscillation induced by the Lorentz force (see 3.1 and 3.2), since, in this case, we may deduce some information concerning the magnetic fields in the invisible convection zone from the flow data. However, at this moment, it is still hard to conclude which one is the correct explanation among the proposed hypotheses described in the previous section. Each of those hypotheses predicts the presence of meridional flow and its solar cycle variation. Detailed helioseismic investigation of meridional flow is hopefully helpful to discriminate among those hypotheses.

\section{Acknowledgements}

I thank Roger Ulrich for giving me permission to reproduce his synoptic plots as Fig. 1, and Sergei Vorontsov and Michael Thompson for offering me their figure as Fig. 2. I also would like to acknowledge helpful discussion with Laurent Gizon.

\section{References}

Antia, H. M. \& Basu, S. 2000 Ap.J. 541, 442-448.

Antia, H. M. \& Basu, S. 2001 Ap.J. 559, L67-L70.

Basu, S. \& Antia, H. M. 2003 Ap.J. 585, 553-565.

Beck, J. G., Gizon, L., \& Duvall, T. L., Jr. 2002 Ap.J. 575, L47-L50.

Blackman, E. G. 2000 Ap.J. 529, 138-145.

Blackman, E. G. \& Chou, T. 1997 Ap.J. 489, L95-L98.

Brandenburg, A. \& Urpin, V. 1998 A. \& Ap. 332, L41-L44.

Covas, E., Tavakol, R., \& Moss, D. 2001 A. \& Ap. 371, 718-730.

Covas, E., Tavakol, R., \& Moss, D. 2004 A. \& Ap. 416, 775-782.

Covas, E., Tavakol, R., Moss, D., \& Tworkowski, A. 2000 A. E Ap. 360, L21-L24. 
Durney, B. R. 2000 Solar Phys. 196, 1-18.

Giles, P. M., Duvall, T. L., Jr., \& Scherrer, P. H. 1998 In Proc. SOHO 6/GONG 98, Structure and dynamics of the interior of the sun and sun-like stars (ed. S. Korzennik \& A. Wilson). pp. 775-780. ESA Publ. Division.

Gizon, L. 2004 Solar Phys. submitted.

Haber, D. A., Hindman, B. W., Toomre, J., Bogart, R. S., Thompson, M. J., \& Hill, F. 2000 Solar Phys. 192, 335-350.

Haber, D. A., Hindman, B. W., Toomre, J., Bogart, R. S., \& Larsen, R. M. 2002 Ap.J. 570, 855-864.

Howard, R. F. 1987 Nature 328, 667-668.

Howard, R. \& LaBonte, B. J. 1980 Ap.J. 239, L33-L36.

Howe, R., Christensen-Dalsgaard, J., Hill, F., Komm, R. W., Larsen, R. M., Schou, J., Thompson, M. J., \& Toomre, J. 2000a Ap.J. 533, L163-L166.

Howe, R., Christensen-Dalsgaard, J., Hill, F., Komm, R. W., Larsen, R. M., Schou, J., Thompson, M. J., \& Toomre, J. 2000b Science 287, 2456-2460.

Itoh, S.-I., Itoh, K., Yoshizawa, A., \& Yokoi, N. 2004 Ap.J. in press.

Kim, E.-J. \& MacGregor, K. B. 2001 Ap.J. 556, L117-L120.

Kim, E.-J. \& MacGregor, K. B. 2003 Ap.J. 588, 645-654.

Kitchatinov, L. L., Pipin, V. V., Makarov, V. I., \& Tlatov, A. G. 1999 Solar Phys. 189, 227-239.

Komm, R., Howe, R., Durney, B. R., \& Hill, F. 2003 Ap.J. 586, 650-662.

Kosovichev, A. G. \& Schou, J. 1997 Ap.J. 482, L207-L210.

Küker, M., Rüdiger, G., \& Pipin, V. V. 1996 A. \& Ap. 312, 615-623.

LaBonte, B. J. \& Howard, R. 1982 Solar Phys. 75, 161-178.

Parker, E. N. 1975 Ap.J. 198, 205-209.

Petrovay, K. \& Forgács-Dajka, E. 2002 Solar Phys. 205, 39-52.

Rüdiger, G. 1989 Differential Rotation and Stellar Convection: Sun and solar-type stars. Gordon and Breach.

Rüdiger, G., Tuominen, I., Krause, F., \& Virtanen, H 1986 A. \& Ap. 166, 306-318.

Schou, J. 1999 Ap.J. 532, L181-L184.

Schou, J. et al. 1998 Ap.J. 505, 390-417.

Schüssler, M. 1981 A. E A Ap. 94, L17-L18.

Snodgrass, H. B. 1985 Ap.J. 291, 339-343.

Snodgrass, H. B. 1991 Ap.J. 383, L85-L87.

Snodgrass, H. B. \& Wilson, P. R. 1987 Nature 328, 696-699.

Spruit, H. C. 2003 Solar Phys. 213, 1-21.

Talon, S., Kumar, P., \& Zahn, J.-P. 2002 Ap.J. 574, L175-L178.

Thompson, M. J. et al. 1996 Science 272, 1300-1305.

Toomre, J., Christensen-Dalsgaard, J., Hill, F., Howe, R., Komm, R., Schou, J., \& Thompson, M. 2003 In Proc. SOHO 12/GONG+ 2002, Local and global helioseismology: the present and future (ed. H. Sawaya-Lacoste). pp. 409-412. ESA Publ. Division.

Ulrich, R. K. 1998 In Proc. IAU Symp. 185, New Eyes to See Inside the Sun and Stars (ed. F.-L. Deubner, J. Christensen-Dalsgaard, \& D. W. Kurtz). pp. 59-72. Kluwer.

Ulrich, R. K. 2001 Ap.J. 560, 466-475.

Ulrich, R. K., Boyden, J. E., Webster, L., Snodgrass, H. B., Padilla, S. P., Gilman, P., \& Shieber, T. 1988 Solar Phys. 117, 291-328.

Vorontsov, S. V., Christensen-Dalsgaard, J., Schou, J., Starkhov, V. N., \& Thompson, M. J. 2002 Science 296, 101-103.

Wolff, C. L. \& Mayr, H. G. 2004 Ap.J. 606, L.163-L166.

Yoshimura, H. 1981 Ap.J. 247, 1102-1112.

Yoshizawa, A. 1990 Phys. Fluids B 2, 1589-1600.

Zhao, J. \& Kosovichev, A. G. 2001 Ap.J. 603, 776-784. 